\title{
Proliferation and differentiation of rat adipose-derived stem cells are regulated by yes-associated protein
}

\author{
XINGZHI JING ${ }^{*}$, JIANG WANG ${ }^{*}$, WEIFENG YIN*, GUANGHUI LI, ZHONG FANG, \\ WENTAO ZHU, FENGJING GUO and YAPING YE
}

\begin{abstract}
Department of Orthopedics, Tongji Hospital, Tongji Medical College, Huazhong University of Science and Technology, Wuhan, Hubei 430030, P.R. China
\end{abstract}

Received December 7, 2017; Accepted June 7, 2018

DOI: $10.3892 /$ ijmm.2018.3734

\begin{abstract}
Adipose-derived stem cell (ASC)-based therapy is a promising treatment strategy for diseases of the musculoskeletal system, as ASCs have the potential to differentiate into numerous cell lineages. However, this field has only recently been explored; therefore, a considerable amount of work is required to determine the therapeutic potential of ASCs. The mechanisms and factors associated with ASC proliferation and differentiation remain to be elucidated. In order to determine the biological properties and subsequent clinical applications of ASCs, these molecular mechanisms must be investigated. The transcriptional co-activator yes-associated protein (YAP), which is a major target of the Hippo signaling pathway, has been reported to serve a crucial role in stem cell proliferation and differentiation. To the best of our knowledge, the role of YAP in the proliferation and differentiation of rat ASCs (rASCs) has not yet been reported. The results of an immunofluorescence analysis revealed that subcellular distribution of YAP in rASCs was regulated by cell density and the actin cytoskeleton. Furthermore, western blot analysis demonstrated that YAP protein expression in rASCs was regulated by lysophosphatidic acid and the actin cytoskeleton. In addition, YAP activation promoted the proliferation of rASCs, whereas YAP inactivation promoted osteogenesis and inhibited adipogenesis of rASCs. In conclusion, these findings demonstrated that YAP may regulate the proliferation and differentiation of rASCs. Targeted modulation of YAP in rASCs may therefore increase the therapeutic effect of rASCs in musculoskeletal diseases.
\end{abstract}

Correspondence to: Mr. Yaping Ye, Department of Orthopedics, Tongji Hospital, Tongji Medical College, Huazhong University of Science and Technology, 1095 Jiefang Avenue, Wuhan, Hubei 430030, P.R. China

E-mail: yyportho@hust.edu.cn

${ }^{*}$ Contributed equally

Key words: rat adipose-derived stem cells, yes-associated protein, Hippo signaling pathway, proliferation, differentiation

\section{Introduction}

Mesenchymal stem cells (MSCs) are adult stem cells that have the ability to self-renew and differentiate into various mesodermal cells, including osteoblasts, chondrocytes and adipocytes (1). Populations of MSCs are present in almost every tissue in the body, including bone marrow, adipose tissue, dental pulp and synovial tissue (2-4). Adipose-derived stem cells (ASCs) are MSCs that are present within the adipose tissue, as first described by Zuk et al (5). It has been reported that ASCs are easier to isolate and acquire compared with other resident stem cell populations, and the cell yield is much higher than that of bone marrow-derived MSCs (BMSCs) (6). ASCs have garnered attention in the scientific and medical fields due to their potential clinical applications (7). Numerous ASCs-based clinical trials have been performed over recent years, and it has been suggested that ASCs possess therapeutic potential for the future treatment of various diseases (7). To fully exploit the therapeutic value of ASCs in clinical application, an in-depth understanding of the molecular pathways by which ASCs proliferate and differentiate is essential.

Yes-associated protein (YAP; gene symbol, YAP1) is a key transcriptional co-factor that is regulated by the Hippo signaling pathway (8). YAP acts as a transcriptional co-activator of the TEA domain-containing sequence-specific transcription factor, which regulates the expression of several 'stemness' genes (9). Core components of the Hippo pathway include the kinases MST and LATS (10). Upon activation of the Hippo pathway, MST phosphorylates and activates LATS, which subsequently phosphorylates and inhibits YAP. YAP phosphorylation leads to cytoplasmic retention and degradation by proteasomes (10). Conversely, inhibition of the Hippo pathway results in YAP nuclear retention and activation of transcriptional activity (11). It has previously been reported that sustained YAP expression is associated with liver enlargement and eventual tumorigenesis, thus suggesting an important role for YAP in cell proliferation and tumor formation (12).

The upstream signaling mechanisms that regulate the Hippo signaling pathway remain elusive. A previous study demonstrated that the mechanical properties of the extracellular matrix (ECM), along with cell matrix attachment, may regulate the localization and activity of YAP via a 
process involving the actin cytoskeleton (13). Furthermore, $\mathrm{G}$ protein-coupled receptors and their agonists, including lysophosphatidic acid (LPA) and sphingosine-1-phosphate (S1P), have been revealed to regulate YAP activity via modulating the actin cytoskeleton (14).

YAP and its paralog, transcriptional co-activator with the PDZ binding motif (TAZ), are the main downstream regulators of the Hippo signaling pathway (15). TAZ has been demonstrated to co-activate genes dependent on Runt-related transcription factor 2 (RUNX2), which is the transcriptional regulator of the osteoblastic lineage, while suppressing the transcription of genes dependent on peroxisome proliferator-activated receptor $\gamma$ (PPAR $\gamma)$, which is the master regulator of the adipogenic lineage, in MSCs. Our previous study demonstrated that the phytomolecule icariin may promote the proliferation and osteogenic differentiation of rASCs via the Ras homolog gene family, member A-TAZ signaling pathway (16). YAP and TAZ are often considered to be orthologs of Drosophila Yorkie; however, it has been reported that the differentiation-regulating functions of YAP are not the same as those of TAZ (17).

The present study aimed to evaluate the upstream factors that affect YAP expression and subcellular distribution in rASCs, as well as the role of YAP in rASC proliferation and osteogenic/adipogenic differentiation.

\section{Materials and methods}

Isolation and culture of rASCs. Male Sprague-Dawley rats (age, 6-8 weeks; weight, 200-250 g, n=24) used in the present study were purchased from the Laboratory Animal Center of the Tongji Medical College (Wuhan, China). All rats were kept in ventilated filter-top cages under standard laboratory conditions: 12-h light/dark cycle and a constant temperature of $24^{\circ} \mathrm{C}$ with $60 \%$ humidity. Rats were given ad libitum access to conventional rodent chow and water. All experimental animals were sacrificed via cervical dislocation. Prior to cervical dislocation, rats were anesthetized by intraperitoneal injection of $2 \%$ pentobarbital sodium $(35 \mathrm{mg} / \mathrm{kg}$ body weight). Animal death was confirmed by monitoring the heartbeat and body temperature. The present study was approved by the Experimental Animal Ethics Committee of Tongji Medical College. Briefly, rASCs were isolated, cultured and characterized as described in our previous study (16). Adipose tissues from the epididymis of male Sprague-Dawley rats were harvested, finely minced and digested with $0.1 \%$ type I collagenase (Wuhan Boster Biological Technology, Ltd., Wuhan, China) at a $1: 1$ volume ratio at $37^{\circ} \mathrm{C}$ for $1 \mathrm{~h}$, followed by centrifugation at $100 \mathrm{x}$ g for $10 \mathrm{~min}$ at $37^{\circ} \mathrm{C}$. The supernatant layer was discarded and the remaining cells were collected and resuspended in Dulbecco's modified Eagle's medium/Ham's F-12 (DMEM/F12; Hyclone; GE Healthcare Life Sciences, Logan, UT, USA) supplemented with $10 \%$ fetal bovine serum (FBS; Gibco; Thermo Fisher Scientific, Inc., Waltham, MA, USA). Undigested debris was removed by filtering through a sterile $75-\mathrm{mm}$ nylon mesh. Finally, cells were cultured with DMEM/F12 supplemented with $10 \% \mathrm{FBS}$ and $1 \%$ penicillin-streptomycin at $37^{\circ} \mathrm{C}$ in an atmosphere containing $5 \% \mathrm{CO}_{2}$. Cells at passage 3 were used for subsequent experiments.
Osteogenic and adipogenic differentiation of rASCs. For osteogenic and adipogenic differentiation, ASCs at passage 3 were plated into 6 -well plates at a density of $1 \times 10^{4}$ cells $/ \mathrm{cm}^{2}$ and cultured at $37^{\circ} \mathrm{C}$ in an atmosphere containing $5 \% \mathrm{CO}_{2}$. After $24 \mathrm{~h}$, the cell culture medium was removed and replaced with adipogenic or osteogenic differentiation medium. The adipogenic differentiation medium comprised DMEM supplemented with $10 \%$ FBS, $1 \mu \mathrm{M}$ dexamethasone (Sigma-Aldrich; Merck KGaA, Darmstadt, Germany), $10 \mathrm{mg} / \mathrm{ml}$ insulin (Invitrogen; Thermo Fisher Scientific, Inc.), $0.5 \mathrm{mM}$ methyl-isobutyl-xanthine (Sigma-Aldrich; Merck KGaA) and $100 \mathrm{mM}$ indomethacin (Sigma Aldrich; Merck KGaA). The osteogenic differentiation medium consisted of DMEM supplemented with $10 \%$ FBS, 10 mM $\beta$-glycerophosphate (SigmaAldrich; Merck KGaA), $0.1 \mu \mathrm{M}$ dexamethasone and $0.1 \mathrm{mM}$ ascorbate-2-phosphate (Sigma-Aldrich; Merck KGaA).

Cell treatment. rASCs at passage 3 were seeded at varying densities $\left(1,500\right.$ or 12,000 cells $\left./ \mathrm{cm}^{2}\right)$ and cultured for 2 days in an atmosphere containing $5 \% \mathrm{CO}_{2}$ at $37^{\circ} \mathrm{C}$ with DMEM/F12 supplemented with $10 \%$ FBS. Cells were harvested and the gene expression of CTGF and Ankrd1 was evaluated via reverse transcription-quantitative polymerase chain reaction (RT-qPCR). To evaluate the role of the actin cytoskeleton in YAP activity, cells were seeded at a density of $1,500 \mathrm{cell} / \mathrm{cm}^{2}$ and cultured for 2 days in an atmosphere containing $5 \% \mathrm{CO}_{2}$ at $37^{\circ} \mathrm{C}$ with DMEM/F12 supplemented with $10 \%$ FBS. After treatment with $1 \mu \mathrm{g} / \mathrm{ml}$ Latrunculin B for $30 \mathrm{~min}$, cells were harvested and the expression levels of CTGF and Ankrd1 were evaluated by RT-qPCR.

rASCs were seeded in 6-cm dishes at a density of 1,500 cells $/ \mathrm{cm}^{2}$ and incubated in serum-free DMEM/F12 medium for $24 \mathrm{~h}$ at $37^{\circ} \mathrm{C}$ prior to treatment with various reagents. LPA at $10 \mu \mathrm{mol} / 1$ and $\mathrm{S} 1 \mathrm{P}$ at $10 \mu \mathrm{mol} / 1$ were used in the present study. Latrunculin B (LatB; $1 \mu \mathrm{g} / \mathrm{ml}$ ) was used to disrupt F-actin fiber organization. All reagents were purchased from Sigma-Aldrich (Merck KGaA).

Immunofluorescence staining. To evaluate the effects of contact-inhibited proliferation on YAP subcellular localization, rASCs were plated on coverslips at varying densities $(1,500$ or 12,000 cells $/ \mathrm{cm}^{2}$ ) and cultured for 2 days in an atmosphere containing $5 \% \mathrm{CO}_{2}$ at $37^{\circ} \mathrm{C}$ with DMEM/F12 supplemented with $10 \%$ FBS. To evaluate the effects of the actin cytoskeleton on YAP subcellular localization, cells were seeded on coverslips at a density of 1,500 cells $/ \mathrm{cm}^{2}$ with DMEM/F12 supplemented with $10 \%$ FBS and treated with $1 \mu \mathrm{g} / \mathrm{ml}$ Latrunculin B at $37^{\circ} \mathrm{C}$ for $30 \mathrm{~min}$. Cells were subsequently fixed with $4 \%$ paraformaldehyde for 15 min and permeabilized with $0.1 \%$ Triton X-100 for $10 \mathrm{~min}$ at room temperature. After blocking with $5 \%$ bovine serum albumin (BSA; Wuhan Boster Biological Technology, Ltd.) for $1 \mathrm{~h}$ at $37^{\circ} \mathrm{C}$, slides were incubated with YAP primary antibody (cat. no. 4912; Cell Signaling Technology, Inc., Danvers, MA, USA) diluted with 5\% BSA (dilution 1:200) at $4^{\circ} \mathrm{C}$ overnight. After washing three times with PBS, slides were incubated with Alexa Fluor ${ }^{\circledR}$ 594-labeled donkey anti-rabbit secondary antibodies (cat. no. R37119; Invitrogen; Thermo Fisher Scientific, Inc.; dilution 1:1,000) for $1 \mathrm{~h}$ at $37^{\circ} \mathrm{C}$. For staining of F-actin, cells were incubated with fluorescein isothiocyanate-conjugated phalloidin (cat. no. P5282; 
Sigma-Aldrich; Merck KGaA) $1 \mathrm{~h}$ after blocking at $37^{\circ} \mathrm{C}$. After washing with PBS, cell nuclei were visualized with DAPI for $5 \mathrm{~min}$. To reduce background fluorescence, slides were washed with PBS. Finally, cells were observed under a fluorescence microscope (FV500; Olympus Corporation, Tokyo, Japan).

Western blot analysis. Cells were washed with PBS and treated with lysis buffer containing $50 \mathrm{mM}$ Tris-HCl, $0.1 \mathrm{mM}$ EDTA, $0.1 \%$ Triton X-100 and $1 \mathrm{mM}$ phenylmethylsulfonyl fluoride for $30 \mathrm{~min}$ at $37^{\circ} \mathrm{C}$. The proteins were prepared using commercial bicinchoninic acid kits (Beyotime Institute of Biotechnology, Jiangsu, China) according to the manufacturer's protocols. Aliquots containing equal amounts of protein $(25 \mu \mathrm{g} / \mathrm{lane})$ were separated by SDS-PAGE (7-15\% gradient cross-linked polyacrylamide gels) and were then transferred to polyvinylidene fluoride membranes (EMD Millipore, Billerica, MA, USA). After transfer, membranes were probed overnight at $4^{\circ} \mathrm{C}$ with anti-YAP rabbit polyclonal antibodies (cat. no. 4912; Cell Signaling Technology, Inc.; dilution 1:1,000), anti-RUNX2 rabbit polyclonal antibodies (cat. no. ab23981; Abcam, Cambridge, UK; dilution 1:200), anti-PPAR $\gamma$ rabbit polyclonal antibodies (cat. no. ab59256 Abcam; dilution 1:200) and anti-GAPDH rabbit polyclonal antibodies (cat. no. BA2913; Wuhan Boster Biological Technology, Ltd; dilution 1:200) followed by horseradish peroxidase-conjugated secondary antibodies for $1 \mathrm{~h}$ at $37^{\circ} \mathrm{C}$ (cat. no. BA1054; Wuhan Boster Biological Technology, Ltd.; dilution 1:10,000). Blots were developed by enhanced chemiluminescence (ECL) using Western ECL Substrate kit (Pierce; Thermo Fisher Scientific, Inc.). The intensity of each band was semi-quantified using digital image analysis software (Quantity One, version 4.6; Bio-Rad Laboratories, Inc., Hercules, CA, USA). GAPDH was selected as a protein loading control.

RT-qPCR analysis. For relative mRNA expression analysis, cells in each group were washed with PBS and treated with TRIzol $^{\circledR}$ (Invitrogen; Thermo Fisher Scientific, Inc.) to extract total RNA, according to the manufacturer's protocol. RNA samples were then purified and reverse-transcribed to cDNA using the 1st Strand cDNA Synthesis kit (Toyobo Life Science, Osaka, Japan), as per the manufacturer's protocol. The cDNA samples were then subject to qPCR to determine the expression of various genes. qPCR was performed with cDNA samples in triplicate under the following conditions: $95^{\circ} \mathrm{C}$ for $5 \mathrm{sec}$, followed by 40 cycles at $94^{\circ} \mathrm{C}$ for $20 \mathrm{sec}, 60^{\circ} \mathrm{C}$ for $20 \mathrm{sec}$ and extension at $72^{\circ} \mathrm{C}$ for $10 \mathrm{sec}$. GAPDH was used as an internal control. Primers used in the present study were as follows: Connective tissue growth factor (CTGF), 5'-CGT TAGCCTCGCCTTGGTG-3' (sense) and 5'-GGGAGCCGA AGTCGCAGA-3' (antisense); ankyrin repeat domain 1 (Ankrd1), 5'-CGGCTCTTGATGACCTTCG-3' (sense) and 5'-GCATTCTCCTTGAGGCTGTC-3' (antisense); YAP, 5'-CCCAAGGCTTGACCCTCGT-3' (sense) and 5'-CGT ATTGCCTGCCGAAATAACT-3' (antisense); proliferating cell nuclear antigen (PCNA), 5'-AAGGGCTGAAGATAA TGCTGATAC-3' (sense) and 5'-CATATACGTGCAAAT TCACCAGAT-3' (antisense); RUNX2, 5'-TGGTACTTC GTCAGCGTCCTATC-3' (sense) and 5'-GCTTCCATCAGC GTCAACACC-3' (antisense); PPAR $\gamma$, 5'-ACATCAGTGGGA ATTAAGGCA-3' (sense) and 5'-TCAAAGGAATGGGAG
TGGTC-3' (antisense); and GAPDH, 5'-GGCAAGTTCAAC GGCACAG-3' (sense) and 5'-CGCCAGTAGACTCCACGA CAT-3' (antisense).

Cell Counting kit (CCK)-8 cell proliferation assay. Cells were cultured under various conditions and subsequently seeded into 96-well plates at a density of $2 \times 10^{3}$ cells/well. Cells were divided into three groups: Serum starvation (SS) group (DMEM/F12 medium only), SS + LPA group (DMEM/F12 medium supplemented with $10 \mu \mathrm{mol} / \mathrm{l} \mathrm{LPA}$ ) and SS + short hairpin (sh) RNA-targeting YAP (shYAP) + LPA group (cells transduced with shYAP lentivirus and cultured in DMEM/F12 medium supplemented with $10 \mu \mathrm{mol} / 1 \mathrm{LPA})$. Cells were cultured for 1 , 2 or 3 days at $37^{\circ} \mathrm{C}$ and each sample was assessed for cellular proliferation. A total of $10 \mu \mathrm{l}$ CCK-8 solution (Dojindo Molecular Technologies, Inc., Kumamoto, Japan) was added to each well and incubated at $37^{\circ} \mathrm{C}$ in the dark for $2 \mathrm{~h}$. Absorbance was read on a microplate spectrophotometer (Bio-Rad Laboratories, Inc.) at a wavelength of $490 \mathrm{~nm}$.

Cell cycle distribution analysis. rASCs were seeded in 6 -well plates at a density of 1,500 cells $/ \mathrm{cm}^{2}$ with serum-free $\mathrm{DMEM} / \mathrm{F} 12$ medium at $37^{\circ} \mathrm{C}$. Cell cycle distribution and DNA content were analyzed by flow cytometry. Cells were divided into the following groups: SS group, SS + LPA group, $\mathrm{SS}+\mathrm{S} 1 \mathrm{P}$ group (DMEM/F12 medium supplemented with $10 \mu \mathrm{mol} / 1 \mathrm{~S} 1 \mathrm{P}), \mathrm{SS}+\mathrm{shYAP}+\mathrm{LPA}$ group and $\mathrm{SS}+$ shYAP + S1P group (cells transduced with shYAP lentivirus cultured in DMEM/F12 medium supplemented with $10 \mu \mathrm{mol} / 1 \mathrm{~S} 1 \mathrm{P})$. After 3 days treatment, cultured cells were rinsed with PBS twice and fixed with $70 \%$ cold ethanol at $-20^{\circ} \mathrm{C}$ for $2 \mathrm{~h}$. Fixed cells were treated with RNase A $(50 \mu \mathrm{g} / \mathrm{ml}$; cat. no. R4875; Sigma-Aldrich; Merck KGaA) at $37^{\circ} \mathrm{C}$ for $30 \mathrm{~min}$ and then stained with propidium iodide (PI; $65 \mu \mathrm{g} / \mathrm{ml}$; Sigma-Aldrich; Merck KGaA) in the dark for $30 \mathrm{~min}$ at $37^{\circ} \mathrm{C}$. PI fluorescence of individual nuclei was measured using a flow cytometer (FACSort; BD Biosciences, Franklin Lakes, NJ, USA).

Alkaline phosphatase (ALP) staining. rASCs were seeded in 6-well plates at a density of $1 \times 10^{4}$ cells $/ \mathrm{cm}^{2}$ and cultured at $37^{\circ} \mathrm{C}$ in an atmosphere containing $5 \% \mathrm{CO}_{2}$ in osteogenic medium. Cells were divided into four groups: Control group (DMEM/F12 supplemented with 10\% FBS), Osteo group (cells cultured in osteogenic differentiation medium), Osteo + control shRNA (shControl) group (cells transduced with control lentivirus cultured in osteogenic differentiation medium) and Osteo + shYAP group (cells transduced with shYAP lentivirus cultured in osteogenic differentiation medium). Following 5 days of culture, cells were rinsed with PBS three times and fixed with $4 \%$ paraformaldehyde for $15 \mathrm{~min}$ at $4^{\circ} \mathrm{C}$. Cells were rinsed again with deionized water and stained with naphthol AS-MX phosphate and fast blue RR salt (Sigma-Aldrich; Merck $\mathrm{KGaA}$ ) for $30 \mathrm{~min}$ at $37^{\circ} \mathrm{C}$ in the dark. Excess dye was removed with PBS and photomicrographs were captured under a light microscope.

Oil Red $O$ staining. rASCs were seeded in 6-well plates at a density of $1 \times 10^{4}$ cells $/ \mathrm{cm}^{2}$ and cultured at $37^{\circ} \mathrm{C}$ in an atmosphere containing $5 \% \mathrm{CO}_{2}$ in adipogenic medium. 
Cells were divided into four groups: Control group, Adipo group (cells cultured in adipogenic differentiation medium), Adipo + shControl group (cells transduced with control lentivirus cultured in adipogenic differentiation medium) and Adipo + shYAP group (cells transduced with shYAP lentivirus cultured in adipogenic differentiation medium). Following 5 days of culture, cells were rinsed with PBS three times and fixed with $4 \%$ paraformaldehyde for $15 \mathrm{~min}$ at $4^{\circ} \mathrm{C}$. Cells were rinsed again with deionized water and stained with Oil Red $\mathrm{O}$ for $30 \mathrm{~min}$ at $37^{\circ} \mathrm{C}$. Excess dye was removed with PBS and photomicrographs were captured under a light microscope.

Construction of recombinant lentiviral vectors. Specific restriction sites of YAP (NM_001034002; Shanghai GeneChem Co., Ltd., Shanghai, China) were inserted into a GV118 plasmid (Shanghai GeneChem Co., Ltd.). The target of shYAP\#1 was GGCAATACGGAATATCAAT, whereas that of shYAP\#2 was GACAGTCTTCCTTTGAGAT. Linked products were identified by double digestion, and DNA sequencing confirmed accurate insertion. The lentiviral vector system included three parts: pGC-LV vector, pHelper 1.0 vector and pHelper 2.0 vector. pHelper 1.0 plasmid DNA $(15 \mu \mathrm{g})$, pHelper 2.0 plasmid DNA $(10 \mu \mathrm{g})$ and pGC-LV shYAP were co-transfected into subconfluent $293 \mathrm{~T}$ cells $(80 \%$ confluence) at $37^{\circ} \mathrm{C}$ for $8 \mathrm{~h}$ using Lipofectamine ${ }^{\circledR} 2000$ (Invitrogen; Thermo Fisher Scientific, Inc.). Supernatants were collected by ultracentrifugation at 4,000 x $\mathrm{g}$ for $10 \mathrm{~min}$ at room temperature and the viral pellet was resuspended in Hanks' balanced salt solution. The vector titers were determined by serial dilution. Lentiviral null vectors with scrambled shRNA (sequence: sense, 5'-UUCUCCGAACGUGUCACG UTT-3' and antisense 5'-ACGUGACACGUUCGGAGA ATT-3') (Shanghai GeneChem Co., Ltd.) were used as the shControl group.

Transduction of rASCs with lentiviral vectors. rASCs at the third passage were seeded at the density of $3 \times 10^{4}$ cells/ml in 24-well tissue culture plates and cultured until cells reached $30 \%$ confluence. Following removal of the cell culture medium, rASCs were incubated with DMEM/F12 containing lentivirus (shYAP or shControl) and $5 \mu \mathrm{g} / \mathrm{ml}$ polybrene (Sigma-Aldrich; Merck KGaA) for $5 \mathrm{~h}$ at $37^{\circ} \mathrm{C}$. Multiplicity of infection (MOI) ranges between 10 and 200 were calculated. Following lentiviral vector infection, the medium was replaced with normal growth medium comprised of DMEM/F12 and 10\% FBS. A total of 1 day post-transduction, reporter gene expression [green fluorescent protein (GFP)] was examined with fluorescence microscopy. ASCs were transduced with lentiviruses at a MOI of 100 and passaged for further study.

Statistical analysis. All data are presented as the means \pm standard deviation. SPSS 13.0 was used for general statistical analysis (SPSS, Inc., Chicago, IL, USA). Significant differences in numerical data between two groups were determined using a Student's t-test. For multiple group comparisons, one-way analysis of variance was used with the Bonferroni post-hoc test. $\mathrm{P}<0.05$ was considered to indicate a statistically significant difference.

\section{Results}

YAP subcellular distribution and expression in rASCs is regulated by cell density and the actin cytoskeleton. YAP is a transcriptional co-activator, which has been reported to serve critical roles in cell proliferation and differentiation; however, the functions of YAP and its upstream regulating factors in rASCs have yet to be elucidated. The present study demonstrated that the subcellular distribution of YAP in rASCs was regulated by cell density and F-actin integrity. rASCs were seeded at varying densities in tissue culture plates $\left(1,500\right.$ and 12,000 cells $\left./ \mathrm{cm}^{2}\right)$. When cultured at 1,500 cells $/ \mathrm{cm}^{2}$ (low density), cells showed no contact with neighboring cells (Fig. 1A). When cultured at a higher density of 12,000 cells $/ \mathrm{cm}^{2}$ (high density), a marked amount of contact was observed with neighboring cells (Fig. 1B). In addition, in the low density group, YAP expression was mainly localized to the nuclei of rASCs (Fig. 1A). Conversely, nuclear YAP expression was markedly decreased when rASCs were cultured at a higher density (Fig. 1B). In addition, the effects of the actin cytoskeleton on YAP subcellular distribution were investigated. The results demonstrated that under normal conditions, YAP was mainly localized in the nuclei of rASCs (Fig. 2A); however, disruption of the actin cytoskeleton with $1 \mu \mathrm{g} / \mathrm{ml}$ LatB for 30 min induced marked YAP cytoplasmic translocation (Fig. 2B).

RT-qPCR was conducted to analyze the expression levels of the YAP target genes, CTGF and Ankrd1. The results indicated that the mRNA expression levels of CTGF and Ankrd1 were significantly decreased in the high density group compared with in the low density group, which was consistent with decreased YAP nuclear localization (Fig. 3A). Furthermore, CTGF and Ankrd1 mRNA expression was markedly inhibited by LatB treatment compared with in the control group, thus suggesting that the integrity of the actin cytoskeleton may be closely associated with YAP activity (Fig. 3B).

$L P A$ promotes YAP protein expression in rASCs. It has previously been reported that LPA activates YAP expression in epithelial cells, such as MCF10A cells (14). However, to the best of our knowledge, the effects of LPA on YAP protein expression in rASCs have yet to be determined. The present study demonstrated that SS of rASCs for $24 \mathrm{~h}$ markedly decreased YAP protein expression (Fig. 4). However, when cells were treated with LPA $(10 \mu \mathrm{mol} / \mathrm{l})$ for $1 \mathrm{~h}$ after $24 \mathrm{~h} \mathrm{SS}$, YAP protein expression was significantly increased (Fig. 4). When cells were treated with the actin cytoskeleton disruption agent, LatB, for $1 \mathrm{~h}$ after $24 \mathrm{~h} \mathrm{SS}$ treatment, YAP protein expression was further decreased compared with in the SS group (Fig. 4). Notably, the LPA-induced upregulation of YAP protein expression in rASCs was inhibited in the SS + LPA + LatB group compared with in the SS + LPA group (Fig. 4). These results suggested that LPA may promote YAP protein expression in rASCs, and that integrity of the actin cytoskeleton is critical for the regulation of YAP protein by LPA.

Transduction of rASCs with the shYAP lentiviral system. rASCs at passage three were infected with lentiviruses carrying shYAP-GFP or shControl-GFP. Fluorescence microscopy was used to detect GFP expression in transduced 

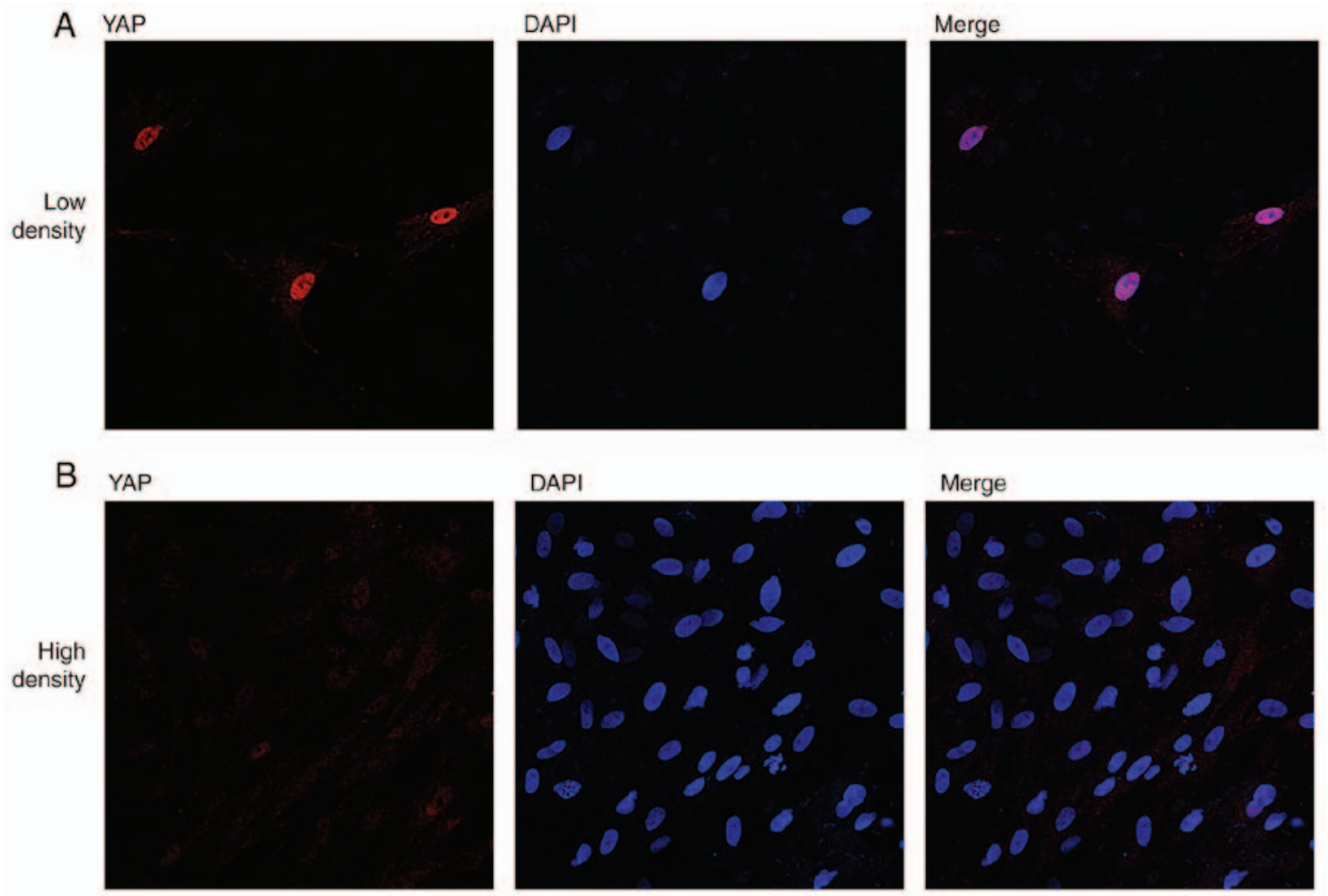

Figure 1. YAP subcellular distribution in rASCs is regulated by cell density. YAP subcellular localization was determined by immunofluorescence staining (red) DNA was stained with DAPI (blue). Magnification, $x 400$. Data are representative of at least three independent experiments. (A) Low density $\left(1,500 \mathrm{cells} / \mathrm{cm}^{2}\right)$ and (B) high density $\left(12,000\right.$ cells/ $\left./ \mathrm{cm}^{2}\right)$ culture groups. rASCs, rat adipose-derived stem cells; YAP, yes-associated protein.

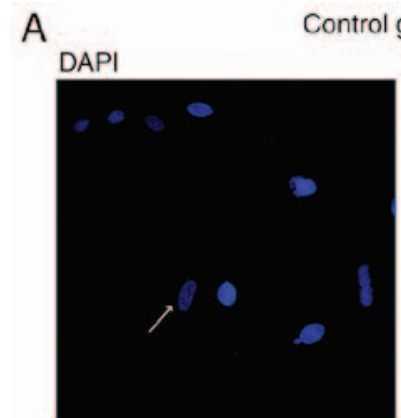

YAP

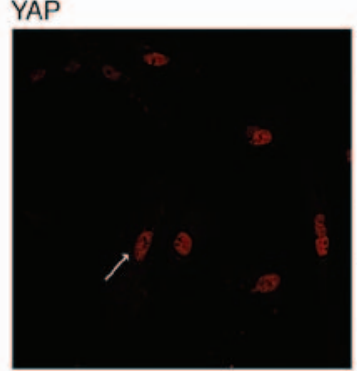

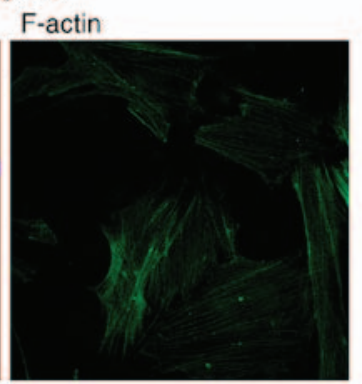

Merge

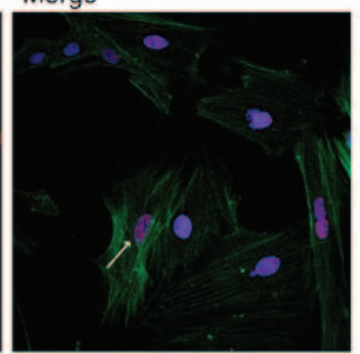

$\mathrm{B}$

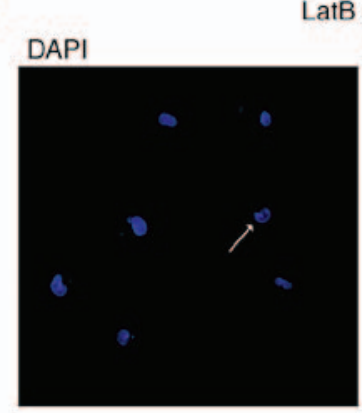

YAP

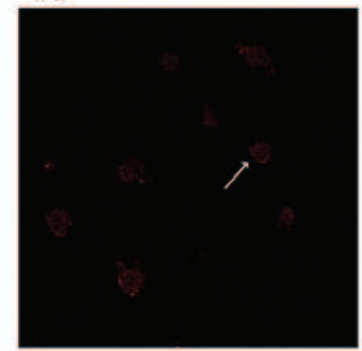

LatB group

\section{F-actin}

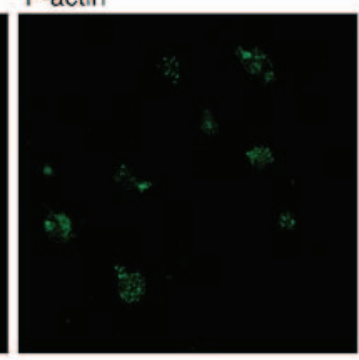

Merge

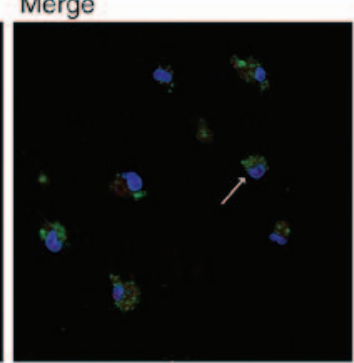

Figure 2. YAP subcellular distribution in rASCs is regulated by the actin cytoskeleton. YAP subcellular localization was determined by immunofluorescence staining (red). DNA was stained with DAPI (blue) and F-actin was stained with fluorescein isothiocyanate-conjugated phalloidin (green). Data are representative of at least three independent experiments. (A) Control group, which contains rASCs without LatB treatment. (B) LatB group, which contains rASCs treated with LatB (1 $\mu \mathrm{g} / \mathrm{ml})$ for 30 min. Magnification, x400; arrows indicate cell nuclei. LatB, latrunculin B; rASCs, rat adipose-derived stem cells; YAP, yes-associated protein.

rASCs. GFP-expressing rASCs were observed by fluorescence microscopy $24 \mathrm{~h}$ post-transduction (Fig. 5A-C). To verify that the lentiviral system had been successfully infected into rASCs, the mRNA expression levels of YAP were measured by
RT-qPCR 5 days post-transduction. The results revealed that YAP mRNA expression in the shYAP\#1 and shYAP\#2 groups was significantly lower than in the shControl group (Fig. 5D). Furthermore, YAP protein expression in the shYAP\#1 and 

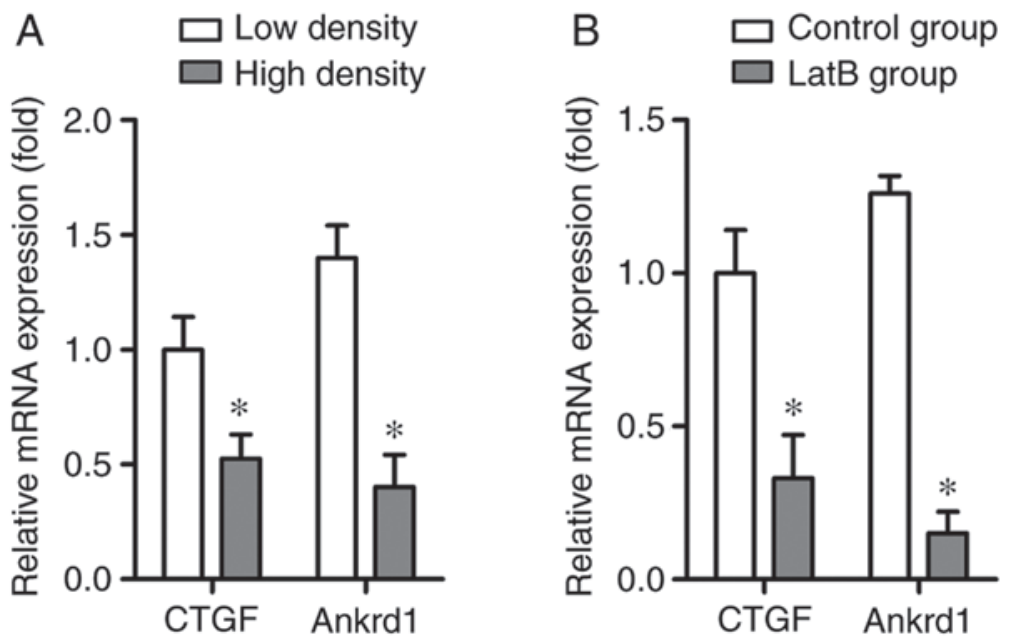

Figure 3. Expression levels of yes-associated protein target genes CTGF and Ankrd1, in rat adipose-derived stem cells are regulated by cell density and the actin cytoskeleton. (A) CTGF and Ankrd1 mRNA expression in the low and high density culture groups. (B) CTGF and Ankrd1 mRNA expression in the Control and LatB groups. Data are presented as the means \pm standard deviation, $\mathrm{n}=3$. ${ }^{*} \mathrm{P}<0.05$ vs. the low density or control groups. Ankrd1, ankyrin repeat domain 1; CTGF, connective tissue growth factor; LatB, latrunculin B.
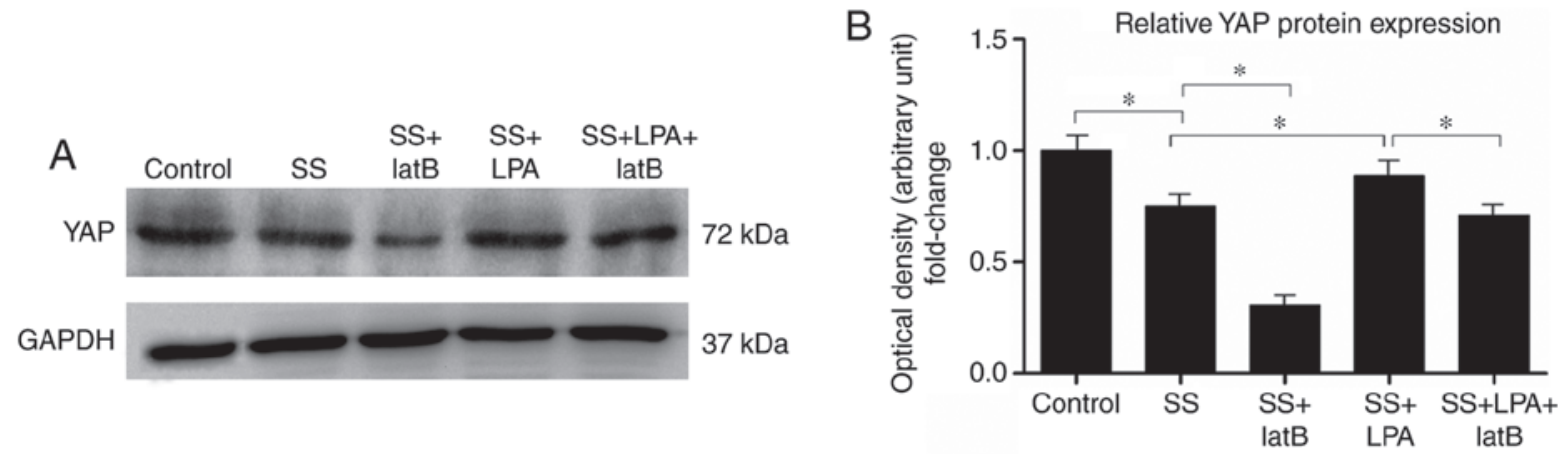

Figure 4. YAP protein expression in rat adipose-derived stem cells is regulated by LPA and the actin cytoskeleton. (A) YAP protein expression was analyzed by western blotting. (B) Histogram represents the average normalized densitometric values from three independent samples. GAPDH was used as the internal control. Data are presented as the means \pm standard deviation, $n=3$. "P<0.05. LatB, latrunculin B; LPA, lysophosphatidic acid; SS, serum starvation; YAP, yes-associated protein.

shYAP\#2 groups was markedly lower compared with in the shControl group 7 days post-transduction (Fig. 5E). The shYAP\#1 lentiviral system was used for subsequent experiments.

LPA and SIP promote rASC proliferation by activating $Y A P$. YAP is a transcriptional coactivator that promotes the expression of various downstream genes, including CTGF and Ankrd1 (14). The present results demonstrated that CTGF, Ankrd1 and YAP mRNA expression levels were significantly increased when rASCs were treated with LPA $(10 \mu \mathrm{mol} / \mathrm{l})$ or S1P $(10 \mu \mathrm{mol} / \mathrm{l})$ for $1 \mathrm{~h}$ compared with in the SS control group (Fig. 6A-C). However, rASCs transduced with shYAP lentiviruses exhibited no increase in CTGF, Ankrd1 and YAP mRNA expression when treated with LPA or S1P (Fig. 6A-C). Furthermore, the expression levels of PCNA in rASCs were evaluated following treatment with LPA and S1P for 3 days. The results demonstrated that LPA and S1P significantly increased PCNA mRNA expression in rASCs (Fig. 6D). However, when rASCs were transduced with a shYAP lentivirus, the effects of LPA and S1P on PCNA expression were abrogated (Fig. 6D).
rASC proliferation was investigated using CCK-8 cell proliferation assays and flow cytometric analysis. CCK-8 results revealed that LPA and S1P significantly increased rASC proliferation compared with in the SS control group 2 and 3 days after treatment (Fig. 6E and F). Infection with a shYAP lentivirus significantly inhibited LPA and S1P-induced cell proliferation (Fig. 6E and F). Cell cycle distribution of rASCs was also investigated using flow cytometry, and the representative flow cytometric profile is presented. Flow cytometric analysis revealed that treating rASCs with LPA and S1P for 3 days significantly increased the percentage of rASCs in $\mathrm{S}$ and $\mathrm{G}_{2} / \mathrm{M}$ phases compared with in the SS control group (Fig. 6G and H). However, when rASCs were infected with a shYAP lentivirus, no significant difference was observed in the percentage of rASCs in the $\mathrm{S}$ and G2/M phases compared with in the SS control group (Fig. 6G and H). In conclusion, these results suggested that LPA and S1P may induce rASC proliferation by activating YAP expression.

YAP inactivation promotes osteogenesis and inhibits adipogenesis of rASCs. The protein expression levels of YAP were 

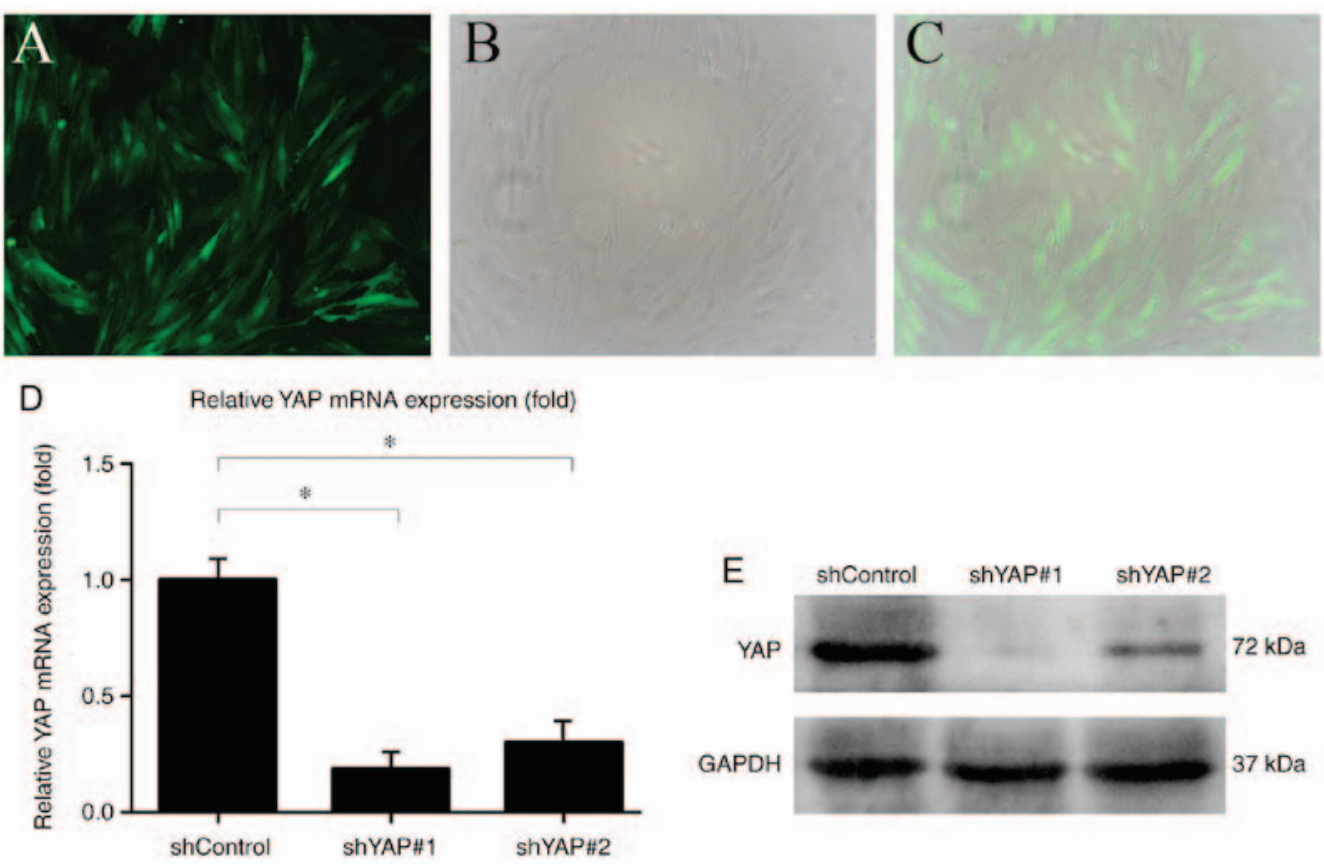

Figure 5. Transduction of shYAP lentiviral system into rASCs. (A) Green fluorescent protein-expressing rASCs were observed under a fluorescence microscope $24 \mathrm{~h}$ after shControl lentiviral transduction. Magnification, $\mathrm{x} 100$. (B) Green fluorescent protein-expressing rASCs were observed under a light microscope $24 \mathrm{~h}$ after shControl lentiviral transduction. Magnification, x100. (C) Merged image of A and B. Magnification, x100. (D) Relative mRNA expression levels of YAP, as determined by reverse transcription-quantitative polymerase chain reaction 5 days after lentiviral transduction. (E) YAP protein expression, as analyzed by western blotting 7 days after lentiviral transduction. Data are presented as the means \pm standard deviation, $\mathrm{n}=3$. $\mathrm{P}<0.05$. rASCs, rat adipose-derived stem cells; sh, short hairpin RNA; YAP, yes-associated protein.
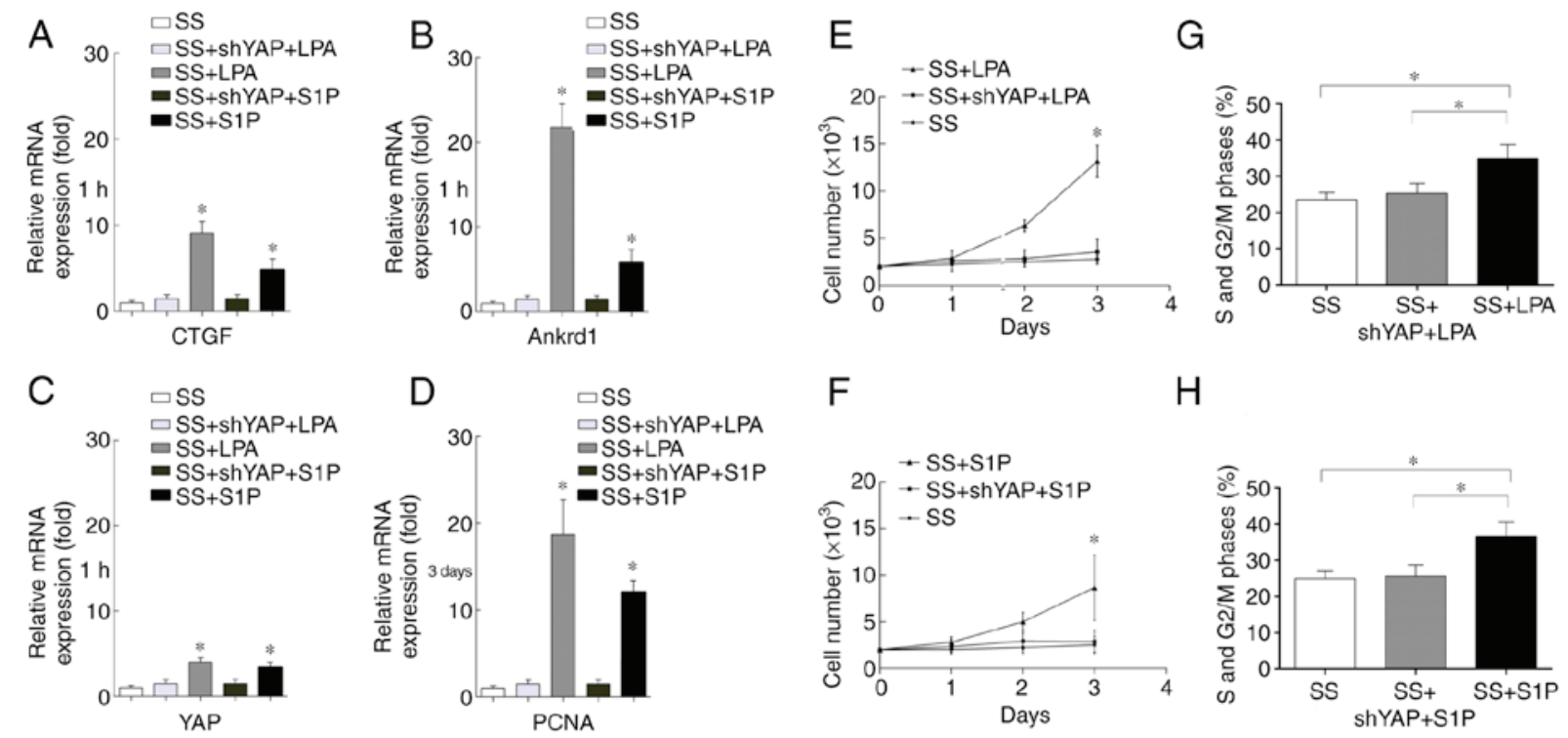

F

$\mathrm{H}$
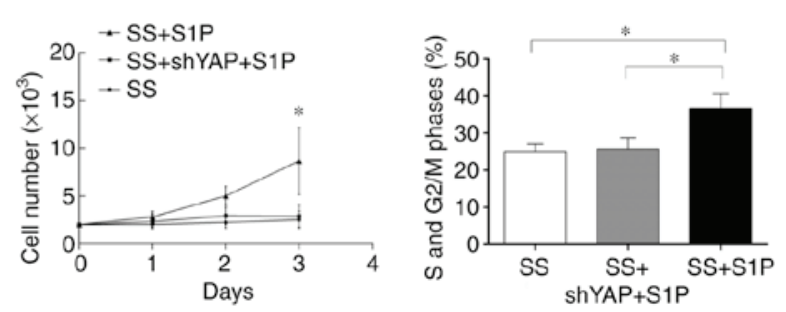

Figure 6. YAP activation promotes the proliferation of rASCs. (A-C) CTGF, Ankrd1 and YAP mRNA expression was assessed $1 \mathrm{~h}$ after LPA or S1P treatment using RT-qPCR. Data are presented as the means \pm standard deviation, $n=3 .{ }^{*} \mathrm{P}<0.05, \mathrm{SS}+\mathrm{LPA}$ vs. SS $+\mathrm{shYAP}+\mathrm{LPA}$ or $\mathrm{SS}+\mathrm{S} 1 \mathrm{P}$ vs. SS + shYAP + S1P. (D) PCNA expression was determined 3 days after LPA or S1P treatment using RT-qPCR. Data are presented as the means \pm standard deviation, $\mathrm{n}=3$. ${ }^{*} \mathrm{P}<0.05, \mathrm{SS}+\mathrm{LPA}$ vs. SS + shYAP + LPA or SS + S1P vs. SS + shYAP + S1P. (E and F) Cell Counting kit- 8 cell proliferation assay. Data are presented as the means \pm standard deviation, $\mathrm{n}=6 .{ }^{*} \mathrm{P}<0.05, \mathrm{SS}+\mathrm{LPA}$ or $\mathrm{SS}+\mathrm{S} 1 \mathrm{P}$ vs. SS. $(\mathrm{G}$ and $\mathrm{H})$ Percentage of rASCs in $\mathrm{S}$ and $\mathrm{G}_{2} / \mathrm{M}$ phases. Data are presented as the means \pm standard deviation, $\mathrm{n}=3 .{ }^{*} \mathrm{P}<0.05$. Ankrd1, ankyrin repeat domain 1 ; CTGF, connective tissue growth factor; LPA, lysophosphatidic acid; PCNA, proliferating cell nuclear antigen; rASCs, rat adipose-derived stem cells; RT-qPCR, reverse transcription-quantitative polymerase chain reaction; sh, short hairpin RNA; SS, serum starvation; YAP, yes-associated protein.

investigated 7 days after osteogenic and adipogenic differentiation of rASCs. The results revealed that YAP protein expression was increased during osteogenic differentiation of rASCs (Fig. 7A and B). Conversely, the protein expression of
YAP was significantly decreased during adipogenic differentiation (Fig. 7A and B).

The present study also investigated the effects of YAP depletion on osteogenic and adipogenic differentiation of rASCs. 
A

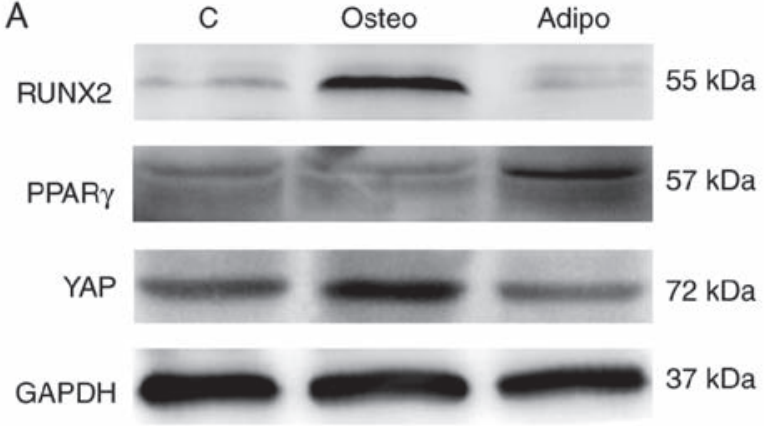

B

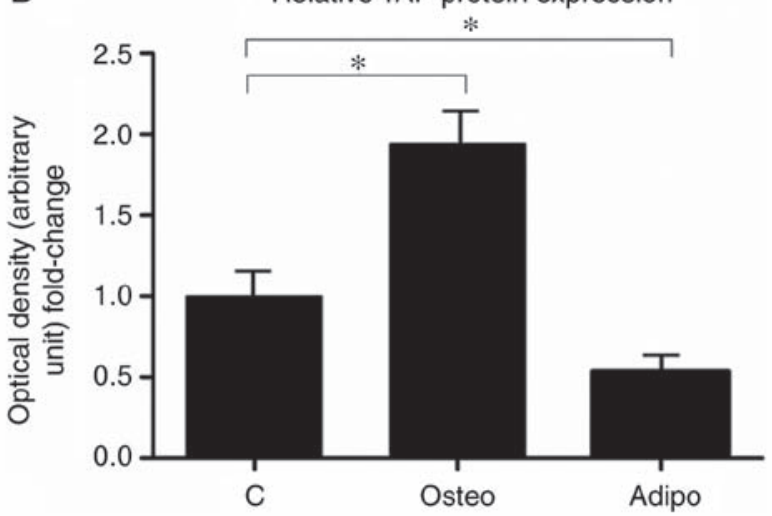

Figure 7. Relative YAP expression 7 days after osteogenesis and adipogenesis of rat adipose-derived stem cells. (A) Relative protein expression was analyzed by western blotting, with GAPDH as an internal control. (B) Histogram represents the average normalized densitometric values from three independent samples. Data are presented as the means \pm standard deviation, $\mathrm{n}=3$. ${ }^{*} \mathrm{P}<0.05$. C, control; PPAR $\gamma$, peroxisome proliferator-activated receptor $\gamma$ RUNX2, Runt-related transcription factor 2; YAP, yes-associated protein.

YAP knockdown following transduction with shYAP led to a marked increase in osteogenic differentiation, as demonstrated by increased ALP staining in the Osteo + shYAP group compared with in the Osteo and Osteo + shControl groups 5 days after cell culture (Fig. 8A). In accordance with this, the expression levels of the osteogenic differentiation-related gene RUNX2 were also increased in the Osteo + shYAP group compared with in the Osteo and Osteo + shControl groups 5 days after cell culture (Fig. 8B). The effects of YAP knockdown on adipogenic differentiation of rASCs were also investigated. The results suggested that adipogenic differentiation of rASCs was reduced following YAP inhibition, as adipocyte formation was markedly decreased 5 days after cell culture in the Adipo + shYAP group compared with in the Adipo and Adipo + shControl groups (Fig. 8C). The adipogenic differentiation-related gene PPAR $\gamma$ was also decreased by YAP knockdown 5 days after cell culture compared with in the control groups (Fig. 8D). In conclusion, these results suggested that YAP may serve an important role in rASCs osteogenic and adipogenic differentiation.

\section{Discussion}

MSCs are promising cells in the field of regenerative medicine. BMSCs and ASCs are two distinct lineages of MSCs, which are currently being investigated due to their potential clinical applications (18). ASCs represent an abundant and easily accessible source of adult stem cells that can differentiate along numerous lineage pathways $(19,20)$. ASC-based therapies for the treatment of musculoskeletal disorders are currently being developed (21); however, the factors that mediate ASC proliferation and differentiation remain poorly understood. Increasing understanding of the molecular mechanisms governing proliferation and differentiation of ASCs may reveal their potential clinical applications.

The regulation of YAP subcellular localization has not been studied extensively. A study by Zhao et al in epithelial cells revealed that YAP localization and phosphorylation are dependent on cell density (22). At low cellular densities, YAP is predominantly localized in the nuclei, translocating to the cytoplasm when cell density is increased (22). Furthermore, Dupont et al reported that YAP/TAZ subcellular localization and activity are regulated by ECM stiffness and cell geometry (23). In addition, the activity of YAP/TAZ in mammary epithelial cells grown on stiff hydrogels has been reported to be comparable to that of cells grown on tissue culture plastic, whereas cells cultured on soft matrices exhibit decreased YAP/TAZ activity (23). It has also been reported that inhibition of the actin cytoskeleton decreases YAP/TAZ nuclear accumulation and transcriptional activity in mammary epithelial cells (23).

The present study demonstrated that the subcellular distribution of YAP in rASCs was regulated by cell density and the actin cytoskeleton. In the low density group, YAP was predominantly localized to the nucleus, whereas in the high density group, YAP expression was markedly decreased in rASC nuclei; however, no significant cytoplasmic localization was detected. These results are not in agreement with those for epithelial cells $(22,23)$. It may be hypothesized that this difference is due to the stem-like characteristics of rASCs and their primordial state, although more thorough investigations are required to confirm this. Consistent with a previous study (23), the present study also indicated that disruption of the actin cytoskeleton with LatB may induce YAP cytoplasmic translocation. The present results suggested that the expression of YAP target genes, CTGF and Ankrd1, was markedly decreased in the high density and LatB-treated groups compared with in the corresponding control groups.

It has previously been reported that LPA acts through G12/13-coupled receptors to inhibit LATS kinase in the Hippo signaling pathway, thereby activating transcriptional co-activators YAP/TAZ (14). In the present study, the effects of LPA on YAP protein expression were detected in rASCs. LPA is a glycerophospholipid-signaling molecule present in all tissues that binds to receptors, such as LPA1-6, to initiate intracellular signaling cascades (24). Because serum contains LPA, in the present study, rASCs were serum starved for $24 \mathrm{~h}$, in order to avoid the effects of LPA in serum on YAP expression. YAP expression was decreased in rASCs cultured in DMEM/F12 (SS without LPA), whereas treatment of rASCs with LatB further decreased YAP expression. Treatment with LPA $(10 \mu \mathrm{mol} / \mathrm{l})$ significantly increased YAP expression, whereas LatB treatment partly abolished the effects of LPA on rASCs. These results suggested that LPA may stimulate YAP expression in rASCs by modulating the actin cytoskeleton. 


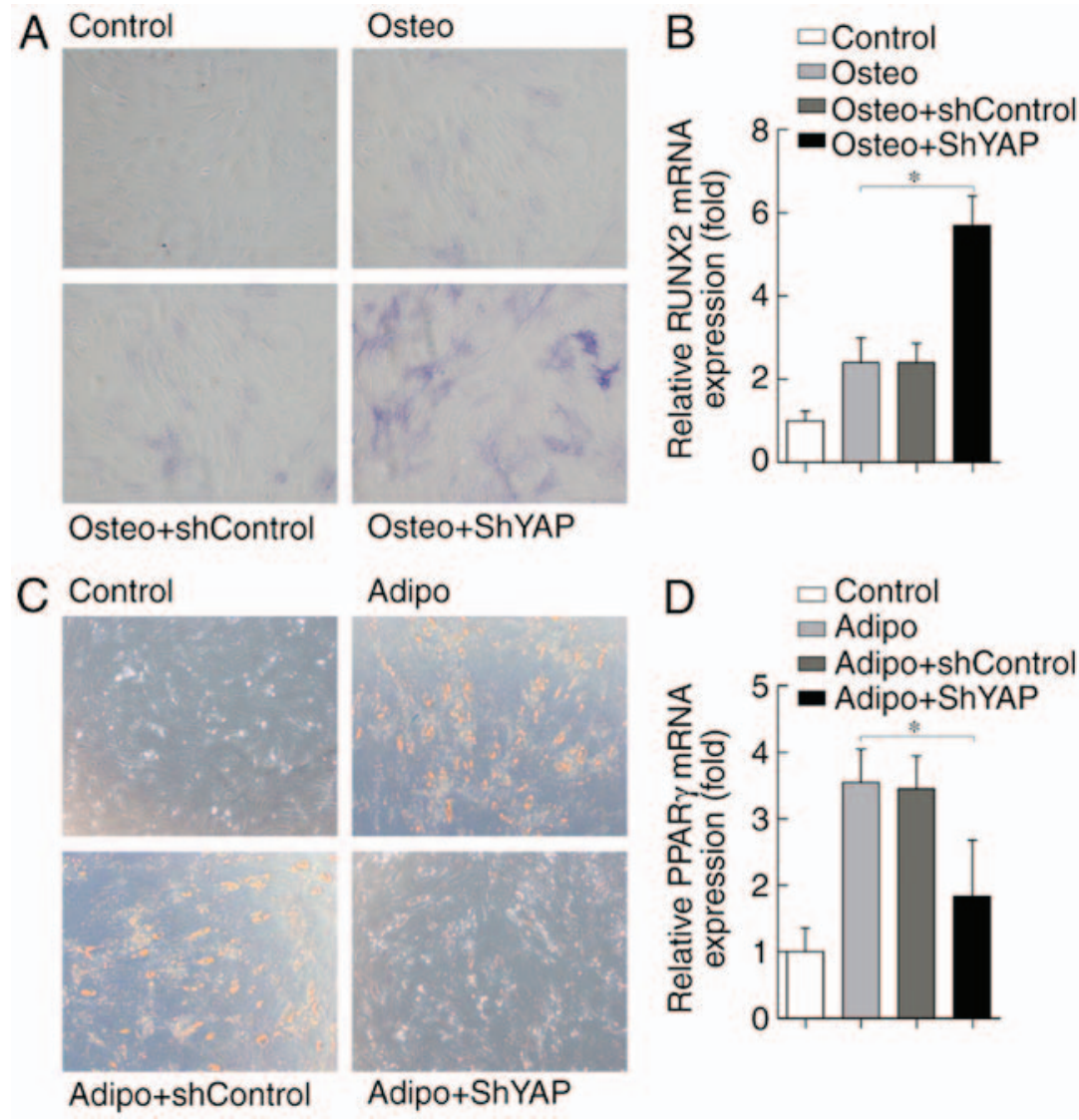

Figure 8. YAP inactivation promotes osteogenesis and inhibits adipogenesis of rASCs. (A) Alkaline phosphatase staining of rASCs 5 days after cell culture. Magnification, x100. (B) Relative expression of RUNX2 mRNA, as determined by RT-qPCR 5 days after cell culture. (C) Oil Red O staining of rASCs 5 days after cell culture. Magnification, $x 100$. (D) Relative expression of PPAR $\gamma$ mRNA, as determined by RT-qPCR 5 days after cell culture. Data are presented as the means \pm standard deviation, $\mathrm{n}=3 .{ }^{*} \mathrm{P}<0.05$. PPAR $\gamma$, peroxisome proliferator-activated receptor $\gamma ;$ rASCs, rat adipose-derived stem cells; RT-qPCR, reverse transcription-quantitative polymerase chain reaction; RUNX2, Runt-related transcription factor 2; sh, short hairpin RNA; YAP, yes-associated protein.

Previous studies have revealed that YAP overexpression increases liver and heart size by increasing cell number (25-27). YAP overexpression in other tissues, such as the skin and intestines, results in an enlargement of the stem cell pool but no overall organ enlargement $(28,29)$. The general conclusion from previous genetic analyses is that YAP induces cell proliferation and tissue development. In the present study, the effects of YAP activation on rASC proliferation were detected. S1P has been reported to possess overlapping effects with LPA (30). The present results revealed that LPA and S1P promoted rASC proliferation, potentially by increasing YAP expression. CTGF and Ankrd1 are both well-characterized YAP target genes. Treatment with LPA and S1P increased CTGF, Ankrd1 and YAP gene expression in rASCs. Furthermore, treatment with LPA or S1P increased the mRNA expression levels of PCNA in rASCs. Conversely, YAP knockdown significantly abrogated the proliferative effects of LPA and S1P on rASCs, thus suggesting that LPA and S1P promote rASC proliferation by activating YAP.

Coordinated proliferation and differentiation of adult stem cells is important for the regeneration and homeostasis of adult tissues. Balanced proliferation and differentiation of muscle satellite stem cells, which express myogenic regulator factor 5, are critical for the innate regeneration response of adult skeletal muscles (31). It has previously been reported that YAP is mainly localized in the nuclei of mouse myoblasts (32), and upon differentiation, YAP is translocated to the cytoplasm and phosphorylated (32). These findings suggested that YAP localization and activity serve a role in the regulation of cell differentiation. The osteogenic differentiation-related master gene RUNX2 has been revealed to interact with YAP and TAZ (33-35). It has also been demonstrated that recruitment of TAZ to RUNX2 target genes significantly promotes osteogenic differentiation of MSCs, whereas TAZ knockdown in MSCs results in reduced osteogenic differentiation (35). To the best of our knowledge, the role of YAP in the osteogenic or adipogenic differentiation of rASCs has yet to be fully elucidated. The present study investigated the effects of YAP inactivation on the osteogenesis and adipogenesis of rASCs. Initially, YAP protein expression was examined during osteogenic and adipogenic differentiation in rASCs; YAP protein expression was increased during osteogenesis and decreased during adipogenesis. Furthermore, YAP knockdown increased osteogenic differentiation and inhibited adipogenic differentiation. Since Wnt signaling is believed to be a major signaling pathway that controls MSCs osteogenic differentiation, it may be hypothesized that YAP blocks osteogenic differentiation induced by Wnt signaling by binding $\beta$-catenin or inducing negative regulators of Wnt signaling. As such, YAP knockdown may enhance osteogenic differentiation, while inhibiting adipogenic differentiation 
of rASCs. These results suggested that YAP is a critical regulator of rASC differentiation; however, to the best of our knowledge, it has only been reported in the present study that YAP protein expression was increased during osteogenesis and decreased during adipogenesis, whereas YAP knockdown increased osteogenic differentiation and inhibited adipogenic defferentiation, thus further studies are required to confirm the detailed molecular mechanisms.

In conclusion, the present study demonstrated that YAP subcellular localization in $\mathrm{rASC}$ s was regulated by cell density and the actin cytoskeleton. Furthermore, it was revealed that YAP expression in rASCs may be regulated, in part, by LPA and the actin cytoskeleton. YAP activation was demonstrated to promote rASC proliferation, whereas YAP knockdown promoted osteogenesis and inhibited adipogenesis. Therefore, targeted modulation of YAP in rASCs may be an effective novel strategy to control rASC proliferation and differentiation for the treatment of various musculoskeletal system diseases. However, the present study is potentially limited by the use of only rat-derived ASCs, and further studies are required using human ASCs, in order to translate these findings into humans.

\section{Acknowledgements}

Not applicable.

\section{Funding}

The present study was supported by grants from the National Natural Science Foundation of China (grant nos. 81702155 and 81371915).

\section{Availability of data and materials}

The data and materials used and/or analyzed during the current study are available from the corresponding author on reasonable request.

\section{Authors' contributions}

$\mathrm{XJ}, \mathrm{YY}$ and FG conceived and designed the experiments. XJ, WY, JW and WZ performed the experiments. WZ and YY analyzed the data. ZF and GL contributed reagents/materials/analysis tools and performed the experiments. XJ and YY wrote the paper. All authors read and approved the final manuscript.

\section{Ethics approval and consent to participate}

The present study was approved by the Experimental Animal Ethics Committee of Tongji Medical College (Wuhan, China).

\section{Patient consent for publication}

Not applicable.

\section{Competing interests}

The authors declare that they have no competing interests.

\section{References}

1. Murphy MB, Moncivais K and Caplan AI: Mesenchymal stem cells: Environmentally responsive therapeutics for regenerative medicine. Exp Mol Med 45: e54, 2013.

2. Davies OG, Cooper PR, Shelton RM, Smith AJ and Scheven BA: A comparison of the in vitro mineralisation and dentinogenic potential of mesenchymal stem cells derived from adipose tissue, bone marrow and dental pulp. J Bone Miner Metab 33: 371-382, 2015.

3. Ye Y, Du Y, Guo F, Gong C, Yang K and Qin L: Comparative study of the osteogenic differentiation capacity of human bone marrow- and human adipose-derived stem cells under cyclic tensile stretch using quantitative analysis. Int J Mol Med 30: 1327-1334, 2012.

4. Qi J, Chen A, You H, Li K, Zhang D and Guo F: Proliferation and chondrogenic differentiation of CD105-positive enriched rat synovium-derived mesenchymal stem cells in three-dimensional porous scaffolds. Biomed Mater 6: 015006, 2011.

5. Zuk PA, Zhu M, Ashjian P, De Ugarte DA, Huang JI, Mizuno H, Alfonso ZC, Fraser JK, Benhaim P and Hedrick MH: Human adipose tissue is a source of multipotent stem cells. Mol Biol Cell 13: 4279-4295, 2002.

6. De Ugarte DA, Morizono K, Elbarbary A, Alfonso Z, Zuk PA, Zhu M, Dragoo JL, Ashjian P, Thomas B, Benhaim P, et al: Comparison of multi-lineage cells from human adipose tissue and bone marrow. Cells Tissues Organs 174: 101-109, 2003.

7. Lim MH, Ong WK and Sugii S: The current landscape of adipose-derived stem cells in clinical applications. Expert Rev Mol Med 16: e8, 2014.

8. Halder G and Johnson RL: Hippo signaling: Growth control and beyond. Development 138: 9-22, 2011.

9. Lian I, Kim J, Okazawa H, Zhao J, Zhao B, Yu J, Chinnaiyan A, Israel MA, Goldstein LS, Abujarour R, et al: The role of YAP transcription coactivator in regulating stem cell self-renewal and differentiation. Genes Dev 24: 1106-1118, 2010.

10. Yu FX, Zhang Y, Park HW, Jewell JL, Chen Q, Deng Y, Pan D, Taylor SS, Lai ZC and Guan KL: Protein kinase A activates the Hippo pathway to modulate cell proliferation and differentiation. Genes Dev 27: 1223-1232, 2013.

11. Zhao B, Tumaneng K and Guan KL: The Hippo pathway in organ size control, tissue regeneration and stem cell self-renewal. Nat Cell Biol 13: 877-883, 2011.

12. Dong J, Feldmann G, Huang J, Wu S, Zhang N, Comerford SA, Gayyed MF, Anders RA, Maitra A and Pan D: Elucidation of a universal size-control mechanism in Drosophila and mammals. Cell 130: 1120-1133, 2007.

13. Zhao B, Li L, Wang L, Wang CY, Yu J and Guan KL: Cell detachment activates the Hippo pathway via cytoskeleton reorganization to induce anoikis. Genes Dev 26: 54-68, 2012.

14. Yu FX, Zhao B, Panupinthu N, Jewell JL, Lian I, Wang LH, Zhao J, Yuan H, Tumaneng K, Li H, et al: Regulation of the Hippo-YAP pathway by G-protein-coupled receptor signaling. Cell 150: 780-791, 2012.

15. Pan D: Hippo signaling in organ size control. Genes Dev 21: 886-897, 2007.

16. Ye Y, Jing X, Li N, Wu Y, Li B and Xu T: Icariin promotes proliferation and osteogenic differentiation of rat adipose-derived stem cells by activating the RhoA-TAZ signaling pathway. Biomed Pharmacother 88: 384-394, 2017.

17. Seo E, Basu-Roy U, Gunaratne PH, Coarfa C, Lim DS, Basilico C and Mansukhani A: SOX2 regulates YAP1 to maintain stemness and determine cell fate in the osteo-adipo lineage. Cell Rep 3: 2075-2087, 2013.

18. Izadpanah R, Trygg C, Patel B, Kriedt C, Dufour J, Gimble JM and Bunnell BA: Biologic properties of mesenchymal stem cells derived from bone marrow and adipose tissue. J Cell Biochem 99: 1285-1297, 2006.

19. Gimble JM, Katz AJ and Bunnell BA: Adipose-derived stem cells for regenerative medicine. Circ Res 100: 1249-1260, 2007.

20. Wang J, Ye Y, Tian H, Yang S, Jin X, Tong W and Zhang Y: In vitro osteogenesis of human adipose-derived stem cells by coculture with human umbilical vein endothelial cells. Biochem Biophys Res Commun 412: 143-149, 2011.

21. Steinert AF, Rackwitz L, Gilbert F, Nöth U and Tuan RS: Concise review: The clinical application of mesenchymal stem cells for musculoskeletal regeneration: Gurrent status and perspectives. Stem Cells Transl Med 1: 237-247, 2012. 
22. Zhao B, Wei X, Li W, Udan RS, Yang Q, Kim J, Xie J, Ikenoue T, Yu J, Li L, et al: Inactivation of YAP oncoprotein by the Hippo pathway is involved in cell contact inhibition and tissue growth control. Genes Dev 21: 2747-2761, 2007.

23. Dupont S, Morsut L, Aragona M,Enzo E, Giulitti S, Cordenonsi M, Zanconato F, Le Digabel J, Forcato M, Bicciato S, et al: Role of YAP/TAZ in mechanotransduction. Nature 474: 179-183, 2011.

24. Choi JW, Herr DR, Noguchi K, Yung YC, Lee CW, Mutoh T, Lin ME, Teo ST, Park KE, Mosley AN, et al: LPA receptors: Subtypes and biological actions. Annu Rev Pharmacol Toxicol 50: 157-186, 2010.

25. Lu L, Li Y, Kim SM, Bossuyt W, Liu P, Qiu Q, Wang Y, Halder G, Finegold MJ, Lee JS and Johnson RL: Hippo signaling is a potent in vivo growth and tumor suppressor pathway in the mammalian liver. Proc Natl Acad Sci USA 107: 1437-1442, 2010.

26. Lee KP, Lee JH, Kim TS, Kim TH, Park HD, Byun JS, Kim MC, Jeong WI, Calvisi DF, Kim JM, et al: The Hippo-Salvador pathway restrains hepatic oval cell proliferation, liver size, and liver tumorigenesis. Proc Natl Acad Sci USA 107: 8248-8253, 2010.

27. Xin M, Kim Y, Sutherland LB, Qi X, McAnally J, Schwartz RJ, Richardson JA, Bassel-Duby R and Olson EN: Regulation of insulin-like growth factor signaling by YAP governs cardiomyocyte proliferation and embryonic heart size. Sci Signal 4: ra70, 2011.

28. Camargo FD, Gokhale S, Johnnidis JB, Fu D, Bell GW, Jaenisch R and Brummelkamp TR: YAP1 increases organ size and expands undifferentiated progenitor cells. Curr Biol 17: 2054-2060, 2007.

29. Zhang H, Pasolli HA and Fuchs E: Yes-associated protein (YAP) transcriptional coactivator functions in balancing growth and differentiation in skin. Proc Natl Acad Sci U S A 108: 2270-2275, 2011.
30. Rosen H, Gonzalez-Cabrera PJ, Sanna MG and Brown S: Sphingosine 1-phosphate receptor signaling. Annu Rev Biochem 78: 743-768, 2009.

31. Tedesco FS, Dellavalle A, Diaz-Manera J, Messina G and Cossu G: Repairing skeletal muscle: Regenerative potential of skeletal muscle stem cells. J Clin Invest 120: 11-19, 2010.

32. Watt KI, Judson R, Medlow P, Reid K, Kurth TB, Burniston JG, Ratkevicius A, De Bari C and Wackerhage $\mathrm{H}$ : Yap is a novel regulator of $\mathrm{C} 2 \mathrm{C} 12$ myogenesis. Biochem Biophys Res Commun 393: 619-624, 2010.

33. Yagi R, Chen LF, Shigesada K, Murakami Y and Ito Y: A WW domain-containing Yes-Associated Protein (YAP) is a novel transcriptional co-activator. EMBO J 18: 2551-2562, 1999.

34. Cui CB, Cooper LF, Yang X, Karsenty G and Aukhil I: Transcriptional coactivation of bone-specific transcription factor Cbfa1 by TAZ. Mol Cell Biol 23: 1004-1013, 2003.

35. Hong JH, Hwang ES, McManus MT, Amsterdam A, Tian Y, Kalmukova R, Mueller E, Benjamin T, Spiegelman BM, Sharp PA, et al: TAZ, a transcriptional modulator of mesenchymal stem cell differentiation. Science 309: 1074-1078, 2005.

This work is licensed under a Creative Commons Attribution-NonCommercial-NoDerivatives 4.0 International (CC BY-NC-ND 4.0) License. 